\title{
Some Probability Characteristics Functions of the Solution of a Stochastic Non-Linear Fredholm Integral Equation of the Second Kind
}

\section{Mohammad Wahdan Muflih *}

Received 1, June, 2010

Accepted 21, June, 2010

\begin{abstract}
:
In this research, some probability characteristics functions (probability density, characteristic, correlation and spectral density) are derived depending upon the smallest variance of the exact solution of supposing stochastic non-linear Fredholm integral equation of the second kind found by Adomian decomposition method (A.D.M)
\end{abstract}

Key words: A.D.M, stochastic non-linear Fredholm integral equation.

\section{Introduction:}

In the beginning of 1980's, a new method for solving linear and nonlinear integral (differential) equations for various kinds has been proposed by G.Adomian, the so called Adomian decomposition method, [1]. After that, in recent years many researchers had been used this method to solve analytically (numerically) either a stochastic Fredholm integral equations or especial kinds of linear (non-linear) Fredholm integral equations [2,3,4]. Most of those researchers are just interested as a final goal in finding the numerical solution on some definite closed interval to study a unique comparison between the numerical solution of a given Fredholm integral equation and its exact solution.

In this paper, our goal is not only interesting in the solution of the supposing stochastic Fredholm integral equation but we concentrate ourself in the derivation of many probability characteristics of this solution (mean, variance, characteristic function, correlation function and spectral density function) that is by depending

*Department of Mathematics-Ibn-Al-Haitham College of Education - University of Baghdad upon the smallest value of the variance of this solution as a basis for that.

So, we consider the following onedimensional non-linear stochastic Fredholm integral equation of the second kind

$$
\mathrm{Y}(\mathrm{w}, \mathrm{t})=\mathrm{X}(\mathrm{w}, \mathrm{t})+\int_{\mathrm{a}}^{\mathrm{b}} \mathrm{k}(\mathrm{t}, \mathrm{s} ; \mathrm{w}) \mathrm{Y}(\mathrm{s}, \mathrm{t}) \mathrm{ds}
$$

where;

(i) $\mathrm{w} \in \Omega, \quad \Omega$ is a sample space sapporting of the probability measure space $(\Omega, F, P)$.

(ii) $\mathrm{Y}(\mathrm{w}, \mathrm{t})$ is the unknown stochastic process for the time $t>0$.

(iii) $\mathrm{X}(\mathrm{w}, \mathrm{t})$ is known stochastic process defined for the time $t>0$.

(iv) $\mathrm{K}(\mathrm{t}, \mathrm{s} ; \mathrm{w})$ is known stochastic kernel defined by $t>0$ and $s \in S$, where $S$ is a compact metric space, $\mathrm{d}$ is any metric defined as $\mathrm{S}$.

(v) $\mathrm{Y}(\mathrm{s}, \mathrm{t})$ is a scalar function defined for the time $t>0, s \in S$. 


\section{Preliminaries}

In equation (1), we consider as a especial case, $\mathrm{X}(\mathrm{w}, \mathrm{t})$ is a standard process with $\mathrm{E}[\mathrm{X}(\mathrm{w}, \mathrm{t})]=0$, $\operatorname{var}[\mathrm{X}(\mathrm{w}, \mathrm{t})]=\mathrm{t}, \mathrm{t}>0,-\infty<\mathrm{w}<\infty$, (i.e.) $X(w, t) \sim N(0, t)$ and $\mathrm{k}(\mathrm{t}, \mathrm{s} ; \mathrm{w})=\mathrm{e}^{-(\mathrm{w}+\mathrm{s}) \mathrm{t}}, 0<\mathrm{s} \leq 1$.

So that (1) becomes,

$$
\mathrm{Y}(\mathrm{w}, \mathrm{t})=\frac{1}{\sqrt{2 \pi \mathrm{t}}} \mathrm{e}^{-\frac{\mathrm{w}^{2}}{2 \mathrm{t}}}+\int_{0}^{1} \mathrm{e}^{-(\mathrm{w}+\mathrm{s}) \mathrm{t}} \mathrm{Y}(\mathrm{s}, \mathrm{t}) \mathrm{ds}
$$

The exact solution of (2) by the Adomian decomposition is [4]

$$
\phi(w, t)=Y_{0}(w, t)+\sum_{n=1}^{\infty} Y_{n}(w, t)
$$

where,

$$
\mathrm{Y}_{0}(\mathrm{w}, \mathrm{t})=\mathrm{X}(\mathrm{w}, \mathrm{t})=\frac{1}{\sqrt{2 \pi \mathrm{t}}} \mathrm{e}^{-\frac{\mathrm{w}^{2}}{2 \mathrm{t}}}
$$

and

$$
\left.\mathrm{Y}_{\mathrm{m}+1}(\mathrm{w}, \mathrm{t})=\int_{0}^{1} \mathrm{k}(\mathrm{t}, \mathrm{s} ; \mathrm{w}) \mathrm{Y}_{\mathrm{m}}(\mathrm{s}, \mathrm{t}) \mathrm{ds}, \mathrm{m}=0,1,2, \ldots\right)
$$

with

$\mathrm{Y}_{0}(\mathrm{~s}, \mathrm{t})=\mathrm{X}(\mathrm{s}, \mathrm{t})=\frac{1}{\sqrt{2 \pi \mathrm{t}}} \mathrm{e}^{-\frac{\mathrm{s}^{2}}{2 \mathrm{t}}}$

$$
\begin{aligned}
Y_{2}(w, t) & =\int_{0}^{1} e^{-(w+s) t} Y_{1}(s, t) d s \\
& =\left[N\left(\frac{1+t^{2}}{\sqrt{t}}\right)-N\left(\frac{t^{2}}{\sqrt{t}}\right)\right] \int_{0}^{1} e^{-(w+s) t} e^{-s t+\frac{t^{3}}{2}} d s \\
& =\left[N\left(\frac{1+t^{2}}{\sqrt{t}}\right)-N\left(\frac{t^{2}}{\sqrt{t}}\right)\right]\left(\frac{1-e^{-2 t}}{2 t}\right) e^{-w t+\frac{t^{3}}{2}},-\infty<w<\infty, t>0
\end{aligned}
$$

For $\mathrm{m}=2$ :

$$
\begin{aligned}
Y_{3}(w, t) & =\int_{0}^{1} e^{-(w+s) t} Y_{2}(s, t) d s \\
& =\left[N\left(\frac{1+t^{2}}{\sqrt{t}}\right)-N\left(\frac{t^{2}}{\sqrt{t}}\right)\right]\left(\frac{1-e^{-2 t}}{2 t}\right) \int_{0}^{1} e^{-(w+s) t} e^{-s t+\frac{t^{3}}{2}} d s \\
& =\left[N\left(\frac{1+t^{2}}{\sqrt{t}}\right)-N\left(\frac{t^{2}}{\sqrt{t}}\right)\right]\left(\frac{1-e^{-2 t}}{2 t}\right)^{2} e^{-w t+\frac{t^{3}}{2}},-\infty<w<\infty, t>0
\end{aligned}
$$

and by repeating for $\mathrm{m}=3,4,5, \ldots$, one can get 
$Y_{k}(w, t)=\left[N\left(\frac{1+t^{2}}{\sqrt{t}}\right)-N\left(\frac{t^{2}}{\sqrt{t}}\right)\right]\left(\frac{1-e^{-2 t}}{2 t}\right)^{k-1} e^{-w t+\frac{t^{3}}{2}}, k=4,5, \ldots$

Therefore, (3) can be written as

$$
\begin{aligned}
\phi(w, t) & =Y_{0}(w, t)+\left[N\left(\frac{1+t^{2}}{\sqrt{t}}\right)-N\left(\frac{t^{2}}{\sqrt{t}}\right)\right] \sum_{n=0}^{\infty}\left(\frac{1-e^{-2 t}}{2 t}\right)^{n} e^{-w t+\frac{t^{3}}{2}} \\
& =Y_{0}(w, t)+\left[N\left(\frac{1+t^{2}}{\sqrt{t}}\right)-N\left(\frac{t^{2}}{\sqrt{t}}\right)\right]\left(\frac{2 t}{e^{-2 t}+2 t-1}\right) e^{-w t+\frac{t^{3}}{2}}
\end{aligned}
$$

Finally, by substituting (4) into above function, the exact solution of (3) over the whole real line will be

$$
\phi(\mathrm{w}, \mathrm{t})=\alpha(\mathrm{t}) \mathrm{e}^{-\frac{\mathrm{w}^{2}}{2 \mathrm{t}}}+\beta(\mathrm{t}) \mathrm{e}^{-\mathrm{wt}},-\infty<\mathrm{w}<\infty, \mathrm{t}>0
$$

where

$$
\alpha(\mathrm{t})=\frac{1}{\sqrt{2 \pi \mathrm{t}}}, \beta(\mathrm{t})=\left[\mathrm{N}\left(\frac{1+\mathrm{t}^{2}}{\sqrt{\mathrm{t}}}\right)-\mathrm{N}\left(\frac{\mathrm{t}^{2}}{\sqrt{\mathrm{t}}}\right)\right]\left(\frac{2 \mathrm{t}}{\mathrm{e}^{-2 \mathrm{t}}+2 \mathrm{t}-1}\right) \mathrm{e}^{\frac{\mathrm{B}^{3}}{2}}
$$

Furthermore, the function $\phi(\mathrm{w}, \mathrm{t})$ can also be considered as a solution over the interval $\quad-1 \leq \mathrm{w} \leq 1$ which permit to derive the probability characteristics of the solution over this interval and over any division of its ten equal subintervals each of length 0.2 starting from $(-1$ to +1$)$ beside choosing many suitable different values for $\mathrm{t} \in \mathrm{T}$ such that

$$
\mathrm{T}=\{0.1,0.4,0.7,1,1.3,1.6,1.9,2.2,
$$

Calculations of $\alpha(\mathrm{t}), \quad \beta(\mathrm{t})$ and $\phi(\mathrm{w}, \mathrm{t})$ are tabulated respectively in tables (1) and (2).

\section{Probability Characteristics:}

\section{Moments, Variances}

The moments and variances of the exact solution (10) are derived over -1 $\leq \mathrm{w} \leq 1$ and for $\mathrm{t}$ which indicating in last section, (i.e.) for $\mathrm{t} \in \mathrm{T}$

$$
\begin{aligned}
\mathrm{E}[\phi(\mathrm{w}, \mathrm{t})] & =\alpha(\mathrm{t}) \int_{-1}^{1} \mathrm{w} \mathrm{e}^{-\frac{\mathrm{w}^{2}}{2 \mathrm{t}}} \mathrm{dw}+\beta(\mathrm{t}) \int_{-1}^{1} \mathrm{w} \mathrm{e}^{-\mathrm{wt}} \mathrm{dw} \\
& =\beta(\mathrm{t})\left[\frac{(1-\mathrm{t}) \mathrm{e}^{-\mathrm{t}}-(1+\mathrm{t}) \mathrm{e}^{\mathrm{t}}}{\mathrm{t}^{2}}\right], \mathrm{t} \in \mathrm{T} \\
\mathrm{E}\left[\phi\left(\mathrm{w}^{2}, \mathrm{t}\right)\right] & =\alpha(\mathrm{t}) \int_{-1}^{1} \mathrm{w}^{2} \mathrm{e}^{-\frac{\mathrm{w}^{2}}{2 \mathrm{t}}} \mathrm{dw}+\beta(\mathrm{t}) \int_{-1}^{1} \mathrm{w}^{2} \mathrm{e}^{-\mathrm{wt}} \mathrm{dw} \\
& =\alpha(\mathrm{t})\left\{\mathrm{t} \sqrt{2 \pi \mathrm{t}}\left[2 \mathrm{~N}\left(\frac{1}{\sqrt{\mathrm{t}}}\right)-1\right]-2 \mathrm{t} \mathrm{e}^{-\frac{1}{2 t}}\right\}+\beta(\mathrm{t})\left[\left(\frac{1}{\mathrm{t}}-\frac{2}{\mathrm{t}^{2}}+\frac{2}{\mathrm{t}^{3}}\right) \mathrm{e}^{\mathrm{t}}-\left(\frac{1}{\mathrm{t}}+\frac{2}{\mathrm{t}^{2}}+\frac{2}{\mathrm{t}^{3}}\right) \mathrm{e}^{-\mathrm{t}}\right], \mathrm{t} \in \mathrm{T}
\end{aligned}
$$

$$
\begin{aligned}
\phi(w, 1.6) & =\alpha(1.6) \mathrm{e}^{-\frac{\mathrm{w}^{2}}{2(1.6)}}+\beta(1.6) \mathrm{e}^{-1.6 \mathrm{w}} \\
& =0.3153 \mathrm{e}^{-\frac{\mathrm{w}^{2}}{32}}+0.1067 \mathrm{e}^{-1.6 \mathrm{w}},-1 \leq \mathrm{w} \leq 1
\end{aligned}
$$

Moreover this function has the and second moments. Table (3) shows that, the smallest variance of (10) is when $(t=1.6)$. Hence, the solution of the supposing Fredholm integral equation (1) over the interval $-1 \leq \mathrm{w} \leq$ 1 that will be adopted in this paper takes the following form, following two properties

- $\phi(\mathrm{w}, 1.6)>0$

- $\int_{-1}^{1} \phi(\mathrm{w}, 1.6) \mathrm{dw} \square 1$

which means that $\phi(w, 1.6)$ is a p.d.f. of the stochastic process $\mathrm{Y}(\mathrm{w}, \mathrm{t})$ which is

$(*) \mathrm{N}(\cdot)$ is a standard normal distribution. 
defined in (1) just when $-1 \leq \mathrm{w} \leq 1$, $\mathrm{t}$ $=1.6$. (fig. (1) represents the curve of $\phi(\mathrm{w}, 1.6))$.

\section{Characteristic Function of $\phi(w, 1.6)$ :}

The characteristic function of $\phi(\mathrm{w}, 1.6)$ can be found as following, [5]

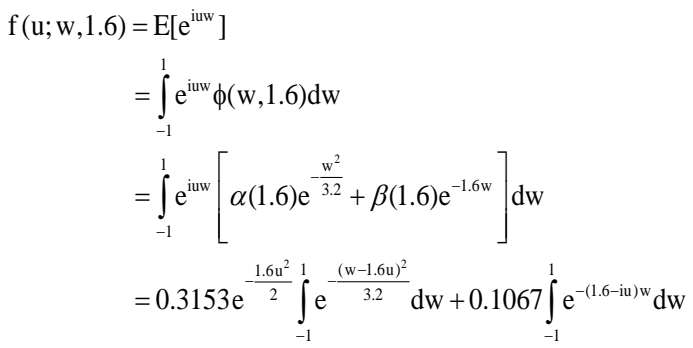

$$
\begin{aligned}
\mathrm{f}(\mathrm{u} ; \mathrm{w}, 1.6) & =0.3153 \sqrt{1.6} \mathrm{e}^{-0.8 \mathrm{u}^{2}} \int_{-1-1.6 \mathrm{u}}^{\frac{1-1.6 \mathrm{uu}}{\sqrt{1.6}}} \mathrm{e}^{-\frac{\mathrm{y}^{2}}{2}} \mathrm{dy}+0.1067 \int_{-1}^{1} \mathrm{e}^{-(1.6-\mathrm{iu}) \mathrm{w}} \mathrm{dw} \\
& =0.3988 \mathrm{e}^{-0.8 \mathrm{u}^{2}} \int_{\frac{-1-1.6 \mathrm{u}}{\sqrt{1.6}}}^{\frac{1-1.6 \mathrm{uu}}{\sqrt{1.6}}}\left[1-\frac{\left(\frac{\mathrm{y}^{2}}{2}\right)}{1 !}+\frac{\left(\frac{\mathrm{y}^{2}}{2}\right)^{2}}{2 !}-\frac{\left(\frac{\mathrm{y}^{2}}{2}\right)^{3}}{3 !}+\ldots\right] \mathrm{dy}+0.1067 \int_{-1}^{1} \mathrm{e}^{-(1.6-\mathrm{iu}) \mathrm{w}} \mathrm{dw} \\
& =0.3988 \mathrm{e}^{-0.8 \mathrm{u}^{2}} \int_{-1}^{\frac{1-1.6 \mathrm{u}}{\sqrt{1.6}}} \sum_{\mathrm{n}=0}^{\infty} \frac{(-1)^{\mathrm{n}} \mathrm{y}^{2 \mathrm{n}}}{(\mathrm{n} !)(2)^{\mathrm{n}}} \mathrm{dy}+0.1067 \int_{-1}^{1} \mathrm{e}^{-(1.6-\mathrm{iu}) \mathrm{w}} \mathrm{dw} \\
& =0.3988 \mathrm{e}^{-0.8 \mathrm{u}^{2}}\left[\left.\sum_{\mathrm{n}=0}^{\infty} \frac{(-1)^{\mathrm{n}} \mathrm{y}^{2 \mathrm{n}}}{(\mathrm{n} !)(2)^{\mathrm{n}}}\right|_{\frac{-1-1.6 \mathrm{u}}{\sqrt{1.6}}} ^{\frac{1-1.6 i u}{\sqrt{1.6}}}\right]-\left.\frac{0.1067}{(1.6-\mathrm{iu})} \mathrm{e}^{-(1.6-\mathrm{iu}) \mathrm{w}}\right|_{-1} ^{1}
\end{aligned}
$$

or

$$
f(u ; w, 1.6)=0.3988 e^{-0.8 u^{2}}\left\{\sum_{n=0}^{\infty} \frac{(-1)^{n}\left[(1-1.6 i u)^{2 n+1}-(-1-1.6 i u)^{2 n+1}\right]}{(n !)(2 n+1)(3.2)^{n}}\right\}+0.1067\left[\frac{e^{(1.6-i u)}-e^{(1.6-i u)}}{(1.6-i u)}\right], u \geq 0 \cdots(
$$

\section{Correlation Function of $\phi(w, 1.6)$ :}

For any $\mathrm{t}_{1}>\mathrm{t}=1.6, \tau=\mathrm{t}_{1}-1.6>$ 0 , the correlation function of $\phi(\mathrm{w}, 1.6)$ with the function $\phi(w, 1.6+\tau)$ depends only on the difference $|\tau|=|-\tau|=\mid \mathrm{t}_{1}-$ 1.6 and can be found as following, [6],

$$
\begin{aligned}
\mathrm{B}(\tau) & =\mathrm{E}(\phi(\mathrm{w}, 1.6) \phi(\mathrm{w}, 1.6+\tau)) \\
& =\mathrm{E}(\phi(\mathrm{w}, 1.6+\tau) \phi(\mathrm{w}, 1.6)) \\
& =\mathrm{B}(-\tau) \\
& =\mathrm{E}(\phi(\mathrm{w}, 1.6) \phi(\mathrm{w}, 1.6+\tau))-\mathrm{E}(\phi(\mathrm{w}, 1.6)
\end{aligned}
$$

$\mathrm{E}(\phi(\mathrm{w}, 1.6+\tau))$

$=\mathrm{E}\left(\phi^{2}(\mathrm{w}, 1.6)\right)+\mathrm{E}(\phi(\mathrm{w}, 1.6) \phi(\mathrm{w}, 1.6+\tau))$

$\mathrm{E}\left(\phi^{2}(\mathrm{w}, 1.6)\right)-\mathrm{E}(\phi(\mathrm{w}, 1.6)) \mathrm{E}(\phi(\mathrm{w}, 1.6+\tau))$ $=\mathrm{E}\left(\phi^{2}(\mathrm{w}, 1.6)\right)+\mathrm{E}(\phi(\mathrm{w}, 1.6) \mathrm{E}(\phi(\mathrm{w}, 1.6+\tau))-$ $(\phi(\mathrm{w}, 1.6))-\mathrm{E}(\phi(\mathrm{w}, 1.6)) \mathrm{E}(\phi(\mathrm{w}, 1.6+\tau))$ $=\mathrm{E}\left(\phi^{2}(\mathrm{w}, 1.6)+\mathrm{E}(\phi(\mathrm{w}, 1.6)[\mathrm{E}(\phi(\mathrm{w}, 1.6+\tau))-\right.$ $\mathrm{E}(\phi(\mathrm{w}, 1.6))]-\mathrm{E}(\phi(\mathrm{w}, 1.6)) \mathrm{E}(\phi(\mathrm{w}, 1.6+\tau))$ $=\mathrm{E}\left(\phi^{2}(\mathrm{w}, 1.6)\right)-\left[\mathrm{E}(\phi(\mathrm{w}, 1.6)]^{2}\right.$

Hence and by table (3)

$\mathrm{B}(\tau)=\operatorname{var}(\phi(\mathrm{w}, 1.6))=0.2887,-1 \leq \mathrm{w} \leq 1, \tau>0$ ...(15)

\section{Spectral Density Function of $\phi(\mathbf{w}, \mathbf{1 . 6})$ :}

The spectral density function of $\phi(w, 1.6)$ for known $\mathrm{B}(\tau)(15)$ can be found by khinchine's formula as following, [6],

$$
\begin{aligned}
\mathrm{f}_{\phi}(\lambda) & =\frac{1}{2 \pi} \int_{-\infty}^{\infty} \mathrm{B}(\tau) \mathrm{e}^{-\mathrm{i} \lambda \tau} \mathrm{d} \tau,|\lambda|<\mathrm{n} \pi, \mathrm{n} \\
=1,2, \ldots & \ldots(16) \\
\mathrm{f}_{\phi}(\lambda) & =\frac{0.2887}{2 \pi} \int_{-\infty}^{\infty}(\cos \lambda \tau-\mathrm{i} \sin \lambda \tau) \mathrm{d} \tau \\
& =\frac{0.2887}{\pi} \int_{-\infty}^{\infty} \cos \lambda \tau \mathrm{d} \tau
\end{aligned}
$$

and for $\tau=\mathrm{t}_{1}-1.6>0$ 


$$
\begin{aligned}
\mathrm{f}_{\phi}(\lambda) & =\frac{0.2887}{\pi} \int_{0}^{\mathrm{t}_{1}-1.6} \cos \lambda \tau \mathrm{d} \tau \\
& =\frac{0.2887}{\pi} \frac{\sin \left(\lambda\left(\mathrm{t}_{1}-1.6\right)\right)}{\lambda} \\
& =0.0919 \frac{\sin \left(\lambda\left(\mathrm{t}_{1}-1.6\right)\right)}{\lambda},|\lambda| \leq \mathrm{n} \pi, \mathrm{n}=1,2 ; \mathrm{t}_{1}>1.6
\end{aligned}
$$

Also,

$$
\mathrm{f}_{\phi}(-\lambda)=0.0919 \frac{\sin \left(-\lambda\left(\mathrm{t}_{1}-1.6\right)\right)}{-\lambda}=\mathrm{f}_{\phi}(\lambda)
$$

which means that $f_{\phi}(\lambda)$ is an even function, [6] and represents the average power in the solution (function) $\phi(w, 1.6)$ at the angular frequency $\lambda$. (Fig.(2) represents the curve of $\mathrm{f}_{\phi}(\lambda)$ for $0 \leq \lambda \leq 2 \pi$ when $\mathrm{t}_{1}=2.6,3.6$ and 11.6).

\section{Conclusions:}

1.For $t>3$, the values of $\beta(t)$ defined in the solution (10) tend rapidly to zero while those for $\alpha(t)$ tend slowly.

2.The solution $\phi(w, t)$ is not a p.d.f. of (10) for all $t \in T$ except for $t=1.6$.

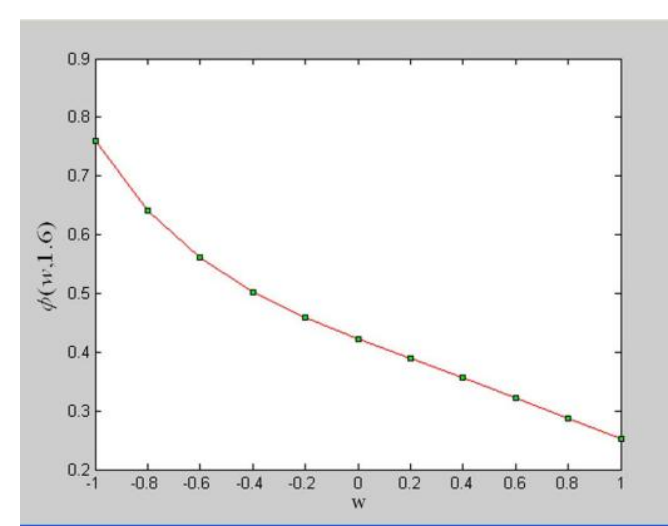

Fig. (1): The curve of $\phi(w, 1.6),-1 \leq w \leq 1$

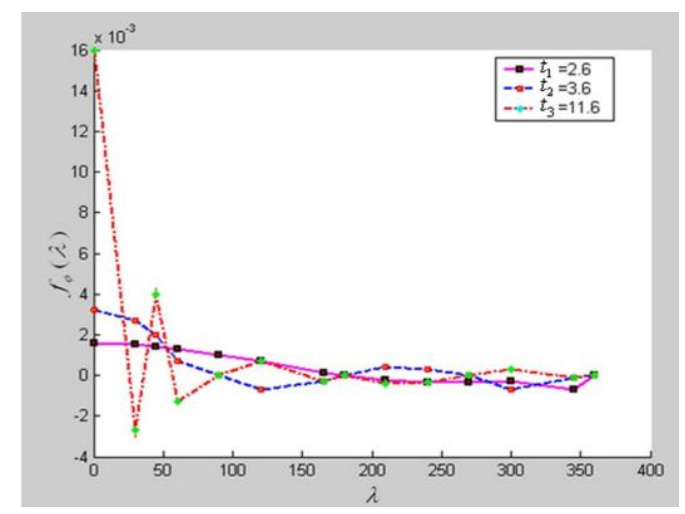

Fig. (2): The curve of $f_{\phi}(\lambda)$ with three different values of $t_{1}, 0<\lambda \leq 2 \pi$

Table (1): $\alpha(\mathrm{t})=\frac{1}{\sqrt{2 \pi \mathrm{t}}}, \beta(\mathrm{t})=\left[\mathrm{N}\left(\frac{1+\mathrm{t}^{2}}{\sqrt{\mathrm{t}}}\right)-\mathrm{N}\left(\frac{\mathrm{t}^{2}}{\sqrt{\mathrm{t}}}\right)\right]\left(\frac{2 \mathrm{t}}{\mathrm{e}^{-2 \mathrm{t}}+2 \mathrm{t}-1}\right) \mathrm{e}^{\frac{\mathrm{t}^{3}}{2}}$
\begin{tabular}{|c|c|c|c|c|c|}
\hline $\mathrm{t}$ & $\alpha(\mathrm{t})$ & $\mathrm{N}\left(\frac{1+\mathrm{t}^{2}}{\sqrt{\mathrm{t}}}\right)-\mathrm{N}\left(\frac{\mathrm{t}^{2}}{\sqrt{\mathrm{t}}}\right)$ & $\frac{2 \mathrm{t}}{\mathrm{e}^{-2 \mathrm{t}}+2 \mathrm{t}-1}$ & $\mathrm{e}^{\frac{\mathrm{t}^{3}}{2}}$ & $\beta(\mathrm{t})$ \\
\hline 0.1 & 1.2614 & 0.4873 & 10.6950 & 1.0005 & 5.2143 \\
\hline 0.4 & 0.6307 & 0.3676 & 10.6776 & 1.0325 & 4.0458 \\
\hline 0.7 & 0.4767 & 0.2450 & 3.2086 & 1.1871 & 0.9332 \\
\hline 1 & 0.3989 & 0.1359 & 2.1652 & 1.6487 & 0.4851 \\
\hline 1.3 & 0.3500 & 0.0603 & 1.7616 & 3.0000 & 0.3187 \\
\hline 1.6 & 0.3153 & 0.0088 & 1.5527 & 7.7524 & 0.1067 \\
\hline 1.9 & 0.2894 & 0.0024 & 1.4281 & 30.6812 & 0.1052 \\
\hline 2.2 & 0.2689 & 0.0002 & 1.3464 & 205.2031 & 0.0552 \\
\hline 2.5 & 0.2523 & 0.00005 & 1.2895 & 2471.3010 & 0.0381 \\
\hline 2.8 & 0.2384 & 0.000005 & 1.2479 & 58454.2690 & 0.0289 \\
\hline
\end{tabular}

Table (2): $\phi(\mathrm{w}, \mathrm{t})=\alpha(\mathrm{t}) \mathrm{e}^{-\frac{\mathrm{w}^{2}}{2 \mathrm{t}}}+\beta(\mathrm{t}) \mathrm{e}^{-\mathrm{wt}}$

\begin{tabular}{|c|c|c|c|c|c|c|c|c|c|c|}
\hline $\mathrm{w}$ & $\mathrm{t}=0.1$ & $\mathrm{t}=0.4$ & $\mathrm{t}=0.7$ & $\mathrm{t}=1$ & $\mathrm{t}=1.3$ & $\mathbf{t}=\mathbf{1 . 6}$ & $\mathrm{t}=1.9$ & $\mathrm{t}=2.2$ & $\mathrm{t}=2.5$ & $\mathrm{t}=2.8$ \\
\hline-1 & 5.7712 & 6.2225 & 2.1126 & 1.5605 & 1.4004 & $\mathbf{0 . 7 5 4 5}$ & 0.6449 & 0.7062 & 0.2467 & 0.2241 \\
\hline-0.8 & 5.7000 & 5.8550 & 1.9355 & 1.3692 & 1.1752 & $\mathbf{0 . 6 4 2 0}$ & 0.5345 & 0.5533 & 0.2464 & 0.2267 \\
\hline-0.6 & 5.7452 & 5.5453 & 1.7889 & 1.2171 & 0.9998 & $\mathbf{0 . 5 6 0 5}$ & 0.4608 & 0.4544 & 0.2495 & 0.2315 \\
\hline-0.4 & 5.9939 & 5.2641 & 1.6599 & 1.0919 & 0.8650 & $\mathbf{0 . 5 0 2 4}$ & 0.4125 & 0.3924 & 0.2533 & 0.2363 \\
\hline-0.2 & 6.4339 & 4.9828 & 1.5367 & 0.9835 & 0.7569 & $\mathbf{0 . 4 5 8 3}$ & 0.3787 & 0.3522 & 0.2557 & 0.2393 \\
\hline 0 & 6.4757 & 4.6765 & 1.4099 & 0.8840 & 0.6685 & $\mathbf{0 . 4 2 2 0}$ & 0.3526 & 0.3241 & 0.2536 & 0.2399 \\
\hline 0.2 & 6.2253 & 4.2510 & 1.2746 & 0.7882 & 0.5902 & $\mathbf{0 . 3 8 8 9}$ & 0.3295 & 0.3020 & 0.2523 & 0.2376 \\
\hline 0.4 & 5.5766 & 4.3346 & 1.1305 & 0.6934 & 0.5184 & $\mathbf{0 . 3 6 5 3}$ & 0.3070 & 0.2822 & 0.2455 & 0.2322 \\
\hline 0.6 & 5.1191 & 3.5847 & 0.9818 & 0.5994 & 0.4507 & $\mathbf{0 . 3 2 2 7}$ & 0.2834 & 0.2625 & 0.2354 & 0.2238 \\
\hline 0.8 & 4.8648 & 3.2213 & 0.8349 & 0.5075 & 0.3861 & $\mathbf{0 . 2 8 7 9}$ & 0.2583 & 0.2420 & 0.2225 & 0.2128 \\
\hline 1 & 4.7266 & 2.8927 & 0.6968 & 0.4204 & 0.3250 & $\mathbf{0 . 2 5 2 2}$ & 0.2319 & 0.2203 & 0.2068 & 0.1995 \\
\hline
\end{tabular}


Table (3):Mean, Variance of $\phi(w, t)$,

\begin{tabular}{|c|c|c|c|}
\hline \multicolumn{4}{|c|}{$-1<w<1$} \\
\hline $\mathrm{t}$ & $\mathrm{E}[\phi(\mathrm{w}, \mathrm{t})]$ & $\mathrm{E}\left[\phi\left(\mathrm{w}^{2}, \mathrm{t}\right)\right]$ & $\operatorname{Var}[\phi(w, t)]$ \\
\hline 0.1 & -0.3129 & 4.2905 & 4.1926 \\
\hline 0.4 & -1.0954 & 6.3630 & 5.1841 \\
\hline 0.7 & -0.4572 & 0.9271 & 0.7181 \\
\hline 1 & -0.3569 & 0.6254 & 0.4980 \\
\hline 1.3 & -0.4073 & 0.5153 & 0.3494 \\
\hline 1.6 & -0.1457 & 0.3099 & 0.2887 \\
\hline 1.9 & -0.1877 & 0.3267 & 0.2915 \\
\hline 2.2 & -0.1276 & 1.4507 & 1.4344 \\
\hline 2.5 & -0.1131 & 0.8881 & 0.8753 \\
\hline 2.8 & -0.1099 & 1.2747 & 1.2626 \\
\hline
\end{tabular}

\section{References:}

1.Adomian, G., 1994, Solving Frontier Problems of Physics, The Decomposition Method, Kluwer, Dordrecht, Holland.

2.Biazar, J. and Ranjbar, A., 2007, A Comparison Between Newton's Method and A.D.M. for Solving
Special Fredholm Integral Equations, IMForum, 5, pp.215-222.

3.Steven J., N., 1996, Spectral and Discrete Approximation to Stochastic Fredholm Integral Equations, M.Sc. Thesis, Texas Technology University, USA.

4.Vahdi, A.R., Mokhtari, M., 2008. On Decomposition Method for System of Linear Fredholm Integral Equations of the Second Kind, App. Math. Sci., 2, pp.57-62.

5.Parzen, E., 1962, Stochastic Processes, Holden-day, 1nc. $1^{\text {st }}$ edition; San Francisco.

6.Basu, A.K., 2003, Introduction to Stochastic Process, Alpha International Ltd. $1^{\text {st }}$ edition, Pangbourne, England.

\section{بعض دوال المزايا الاحتمالية لحل معادلة فريدهولم التكاملية والعثوائية وغير الخطية من النوع معالة فيداني}

محمد وهلان مفلح*

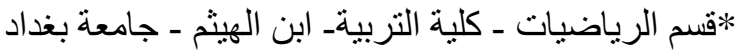

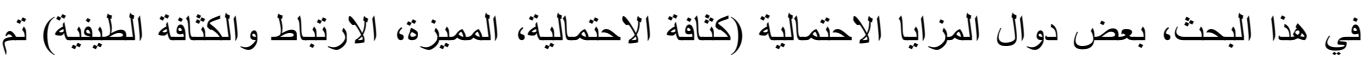

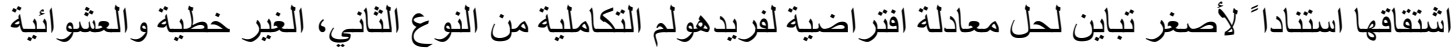
و الذي تم ايجاده بطريقة أدوميان التحليلية. 